\title{
Acute Retinal Necrosis (ARN): Time is Retina (A Case Study)
}

\author{
W. Akioud, T. Abdellaoui, A. Elkhouyali, F. Elasri, Y. Mozari, K. Reda, and A. Oubaaz
}

\section{ABSTRACT}

Acute retinal necrosis (ARN) is an inflammatory condition characterized by a peripheral retinal necrosis due to an infection with varicella-zoster virus or herpes simplex virus. It is commonly seen in immunocompetent adults and is known for its rapid evolution and poor prognosis. Our case study illustrates the gravity of this condition with an extremely rapid ARN that was hard to diagnose.

Keywords: Acute retinal necrosis, infection, herpes, retina.
Submitted : March 24, 2021

Published : April 14, 2021

ISSN: $2593-8339$

DOI: $10.24018 /$ ejmed.2021.3.2.780

\section{W. Akioud*}

Mohammed V Military Teaching Hospital, Rabat, Morocco.

(e-mail: dr.akioudwafae ${ }^{@}$ gmail.com) T. Abdellaoui

Mohammed V Military Teaching

Hospital, Rabat, Morocco.

A. Elkhouyali

Mohammed V Military Teaching Hospital, Rabat, Morocco.

F. Elasri

Mohammed V Military Teaching Hospital, Rabat, Morocco.

Y. Mozari

Mohammed V Military Teaching

Hospital, Rabat, Morocco.

K. Reda

Mohammed V Military Teaching

Hospital, Rabat, Morocco.

A. Oubaaz

Mohammed V Military Teaching

Hospital, Rabat, Morocco.

\section{INTRODUCTION}

Acute Retinal Necrosis syndrome (ARN) is a rare viral disease with a fast evolution.

ARN was first described by Uryama in 1971 [1]. It was associated- few years later- with the Herpes Virus subfamily infection. In 1994 this clinical entity was finally defined as the association of retinal necrosis extending from the periphery to the center of retina, occlusive arterial vascularitis and an anterior segment acute inflammation [2].

The ARN typically concerns young immunocompetent patients, although some cases of ARN during HIV infection were described [3].

The poor prognosis of the ARN as well as its fast evolution makes on the top of the ocular emergencies.

We report a case of a unilateral ARN with an extremely rapid progression, posing as a challenge like no other for our ophthalmological emergency department.

\section{CASE Study}

Mr. A.B is a 39 year old patient with no medical history, who suffered a sudden decreased vision and photophobia of the left eye. Three hours later, the patient consulted a private ophthalmologist who suspected an ARN and referred the patient to our emergency department with a letter mentioning, among the examination elements, a visual acuity of $5 / 10$ of the left eye. It took the patient four hours to get to our department where his ocular exam showed the following elements:

- A normal exam of the right eye with VA of 10/10 and no abnormalities to be mentioned.

- As for the left eye, the VA dropped to hand movements. The slit lamp exam showed an eye redness, thin keratic precipitates, a cellular floaters (1+) and an intraocular pressure of $12 \mathrm{mmHg}$. The Iris has no nodules or structural abnormalities, and a Marcus Gunn sign was found. Posterior segment examination showed a vitreous inflammation, a papillitis, multifocal ischemic areas in the peripheral retina and vascular arteriolitis, as well as a serous retinal detachment (Fig. 1-3). 
- The patient precisely denied any previous ocular surgeries, trauma, oral or genital ulcers, rashes, weight loss, diarrhea, fever, or general fatigue as well as affirming no recent contact with any animal.

- A Fluorescein Angiography was conducted showing extravasation of the dye from vessels wall in the left eye as well as scattered fluorescein blockage (Fig. 4-5).

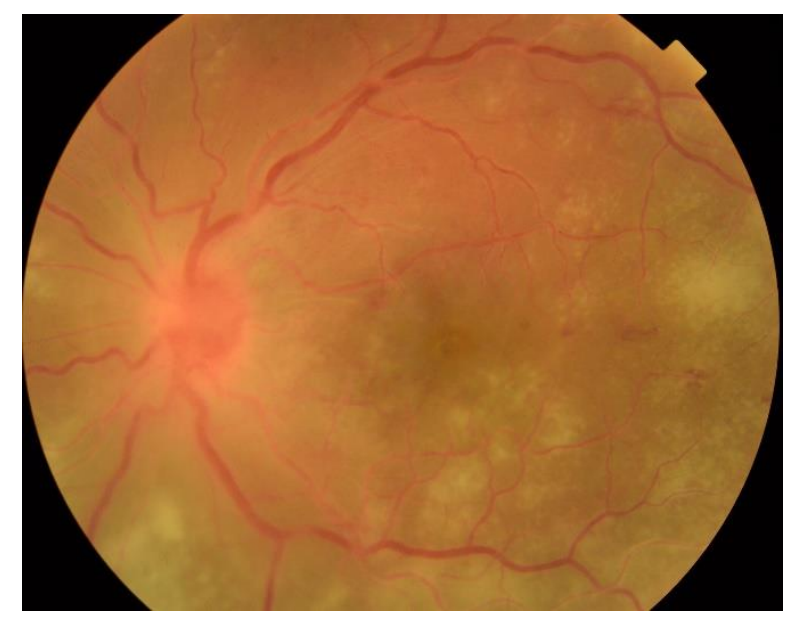

Fig. 1. Fundus showing a papillitis and ischemic areas in the peripheral retina.

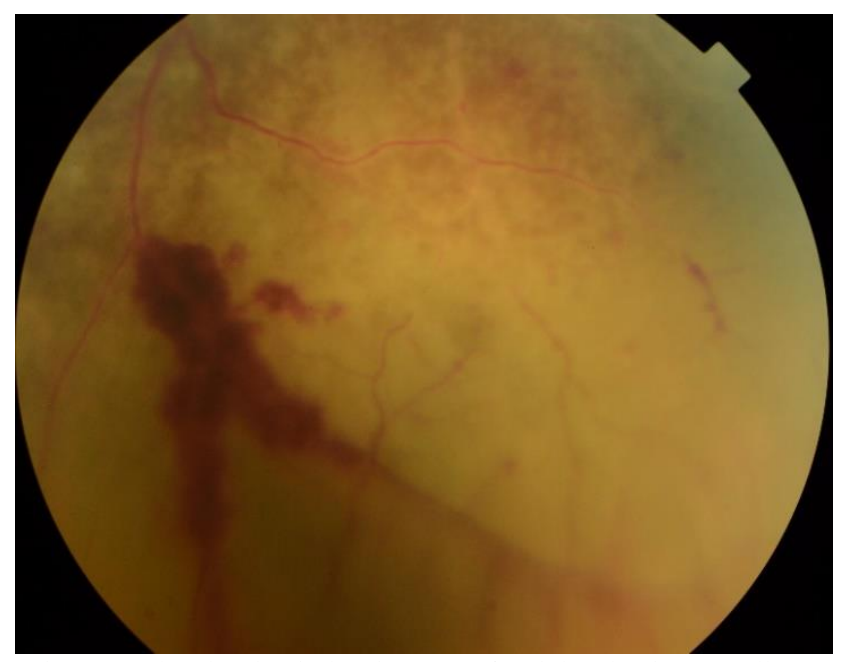

Fig. 2. Fundus showing ischemic zones of peripheral retina as well as a retinal hemorrhage and vascularitis.

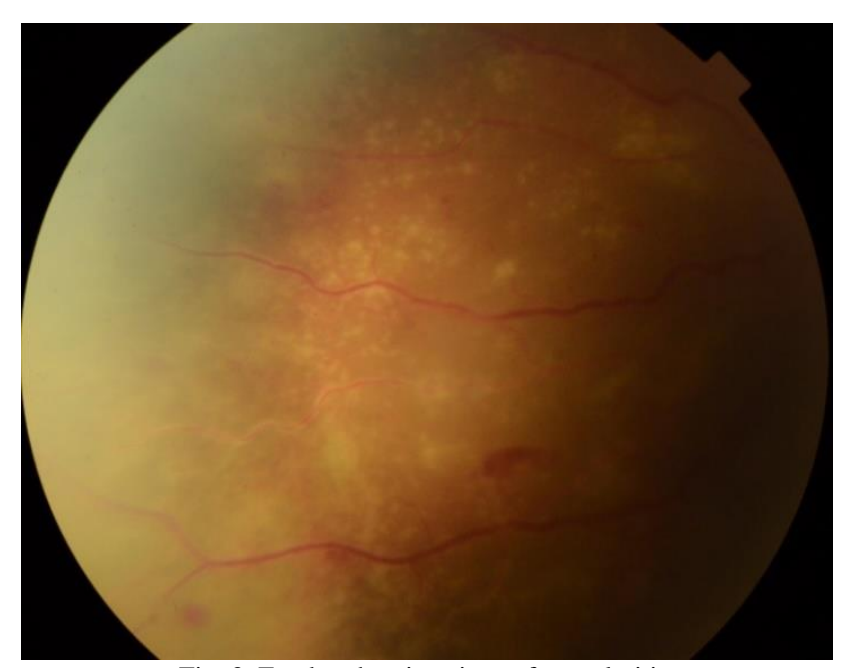

Fig. 3. Fundus showing signs of vascularitis.

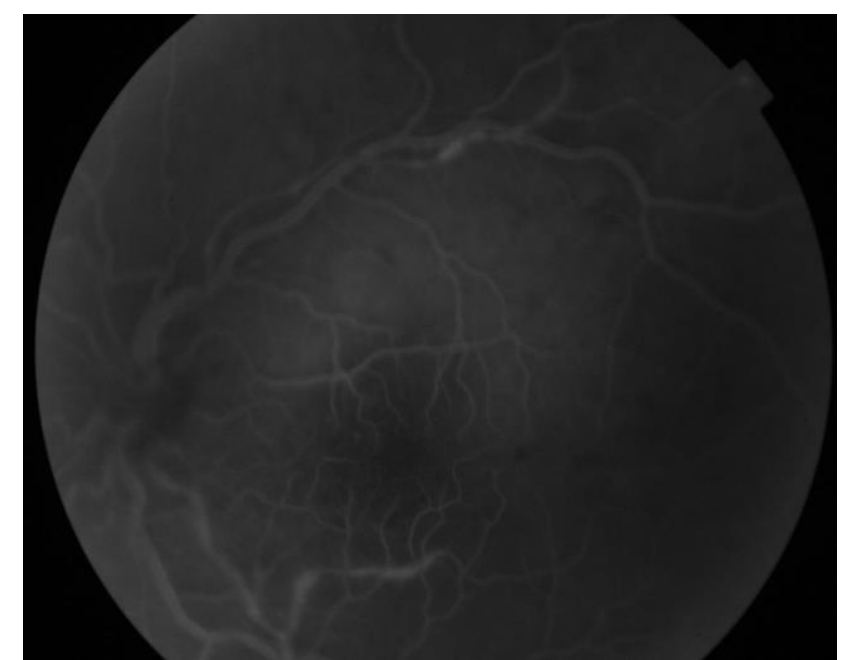

Fig.4. Angiography showing signs of occluded retinal vessels and vascularitis.

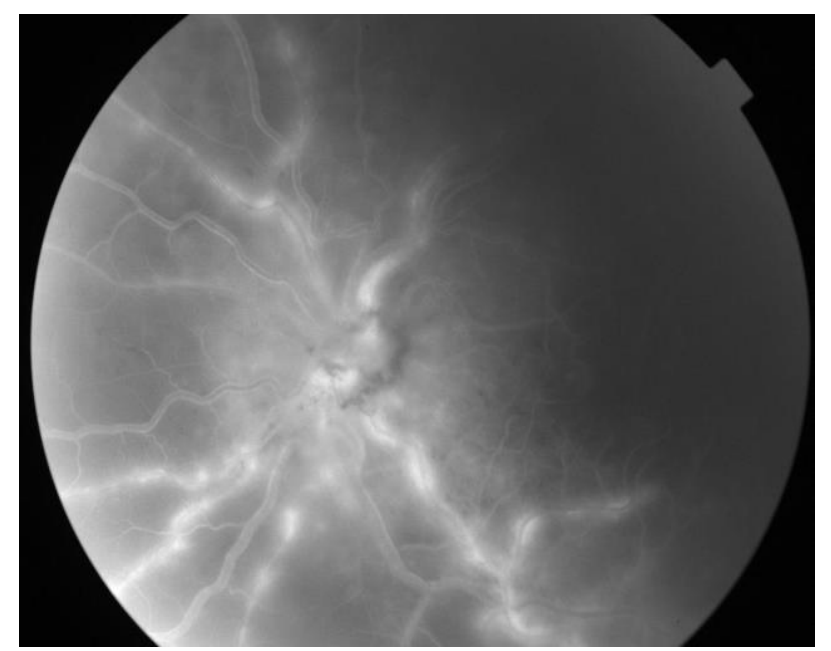

Fig. 5. Angiography showing extravasation of the dye from vessels wall in the left eye as well as scattered fluorescein blockage.

The physical exam was otherwise unremarkable.

Laboratory tests showed normal blood cell counts and normal serum chemistries. Serum was evaluated for Herpes Simplex Virus (HSV), Human Immunodeficiency Virus (HIV), Cytomegalovirus (CMV), Varicella Zoster Virus (VZV) using Polymerase Chain Reaction (PCR) and the results were all negative.

Meanwhile the patient's vision acuity of the left eye dropped to light perception, and he developed a corneal edema as well as a diffuse chemosis.

We conducted immediately a lumber puncture with PCR analysis which came in favor of VZV meningitides. Upon the results the patient stared IV aciclovir (10 mg per Kg every 08 hours) for three weeks which he was transitioned to long-term oral valaciclovir and a bolus of IV steroids (15 mg/KG/day) for three days, then oral steroids for the rest of the treatment period.

In the few next days, the visual acuity of the left eye increased to $1 / 10$, while the vitritis decreased. 


\section{DISCUSSION}

Acute Retinal Necrosis ARN was first describe by Akira Urayama in 1971 [1] as unilateral acute panuveitis associate with retinal periarteritis in a progressive process leading to diffuse necrotizing retinitis and retinal detachment.

In 1994, the American Uveitis Society redefined ARN as a whole of on clinical characteristics and disease course to include [4]:

1) one or more foci of retinal necrosis with discrete borders located in the peripheral retina;

2) rapid progression in the absence of antiviral therapy;

3) circumferential spread;

4) evidence of occlusive vasculopathy with arterial involvement; and

5) a prominent inflammatory reaction in the vitreous and anterior chambers.

ARN is believed to be caused by viral infection. Most commonly the VZV or the HSV. Some case reports suggested the possible association with CMV or Epstein-Barr virus (EBV), although with limited evidence to support these viruses causing the ARN [3].

It often affects healthy immunocompetent adult in their fifth to seventh decade, which was not the case for our midtwenty's patient.

Clinically, in ARN, there are multifocal necrotic areas with discrete borders spreading circumferentially and posteriorly from mid peripheral retina. ARN can present with pain due to iridocyclitis, vitritis, retinal vasculitis, in addition to retinal necrosis.

Anterior segment involvement may include episcleritis, scleritis, corneal edema, keratic precipitates, and anterior chamber inflammation Classically, posterior segment involvement includes vitreous inflammation, retinal vascular arteriolitis, and peripheral retinitis. Retinitis typically begins as multifocal areas of retinal whitening and opacification, oftentimes with scalloped edges; initially, this patchy retinitis usually appears peripherally and, with progression, becomes increasing confluent and more posterior in its location [5].

Arterioles are very much involved, occluded in the fluorescein angiography.

Many Lab tests may be performed in order to identify the virus in cause, including:

- Viral PCR

- Viral culture

- Use of direct or indirect immunofluorescence to identify viral antigens

- Intraocular antibody titers with calculation of the Goldmann-Witmer coefficient

- Vitreous sample to identify the virus directly

These tests were negative in our case study, which made the diagnosis more difficult. The virus was only found in the lumbar puncture fluid making the LP a very important lab test in atypical ARN cases.

Without intervention, the active phase of the disease normally lasts between 6-12 weeks, which was not the case for our patient as the ARN progresses within hours in an excessive rapid way.
Steroids may have a beneficial therapeutic effect if initiated 24-48 hours after the start of antiviral therapy or once regression of retinal necrosis been demonstrated

treatment with antivirals and/or steroids may reduce the latency to 4-6 weeks.

Patients with unilateral ARN should be closely followed with dilated examination of both of their eyes. Approximately $50-75 \%$ of patients with ARN will go on to develop a retinal detachment.

Many complications may occur in the follow up, including:

- Retinal holes and tears;

- Retinal detachment;

- Proliferative vitreoretinopathy;

- Vitreous hemorrhage;

- Macular pucker;

- Optic neuropathy- pallor of the optic disc;

- Encephalitis.

\section{CONCLUSION}

Acute retinal necrosis (ARN) is an inflammatory condition with a poor prognosis, which makes it one of the most urgent ophthalmological emergencies. Because of its fast evolution and numerous complications, it is one of severe condition when "Time is Retina".

\section{REFERENCES}

[1] Urayama AYN, Sasaki Tet al. Unilateral acute uveitis with periarteritis and detechment. Jpn J Ophthalmol 1971; 25: 607-19.

[2] Holland GN and the Executive Commitee of the American Uveitis Society. Standard diagnostic criteria for the acute retinal necrosis syndrome. Am J Ophthalmol, 1994;117:663-6.

[3] Labetoulle M, Offret H, Haut J, Bloch-Michel E, Ullern M, Monin. Le syndrome de nécrose rétinienne aiguë. Etude détrospective à propos de 14 yeux de 11 patients. J Fr Ophtalmol, 1995; 18: 777-87.

[4] Young NJ, Bird AC. Bilateral acute retinal necrosis. Br J Ophthalmol. 1978; 62: 581-590.

[5] Fisher JP, Lewis ML, Blumenkranz M, et al. The acute retinal necrosis syndrome. Part 1: clinical manifestations. Ophthalmology 1982;89(12):1309-16.

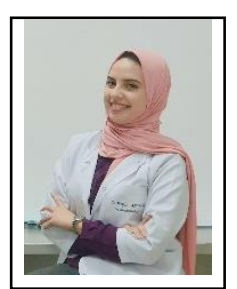

Dr. Wafae Akioud

Senior ophthalmologist at the Military Hospital of Rabat, Morocco.

Owner of the Instagram page: @noweyesee. A simple platform to discuss clinical cases in a funny different way.

Active member of many charity associations in Morocco. 